\title{
Sind ländlich abgelegene Kindertageseinrichtungen ,abgehängte“" Kindertageseinrichtungen? Befunde zu Unterschieden in der Strukturqualität im Stadt-Land-Vergleich am Beispiel Oberfrankens
}

\author{
Doreen Müller
}

Online publiziert: 25 . August 2020

(C) Der/die Autor(en) 2020

Zusammenfassung Der Beitrag beschäftigt sich mit der Frage, ob zwischen städtischen, stadtnahen und ländlich abgelegenen Kindertageseinrichtungen Unterschiede hinsichtlich der Strukturqualität bestehen. Im Gegensatz zu bisherigen Forschungsergebnissen wird dies losgelöst von administrativen Grenzen betrachtet und beleuchtet Besonderheiten kleinräumiger Regionen. Die Ergebnisse basierend auf Daten des Projekts „BildungsLandschaft Oberfranken (BiLO)“ zeigen, trotz Unterschieden zwischen städtischen und ländlich abgelegenen Einrichtungen, keinen deutlichen Hinweis, dass ländlich abgelegene Einrichtungen ,,abgehängte“ Einrichtungen darstellen. Einrichtungen aus dem städtischen Umland, die administrativ der Landregion angehören, sind mal den städtischen mal den ländlich abgelegenen Einrichtungen ähnlich.

Schlüsselwörter Kindergartenqualität · Strukturqualität · Regionale Unterschiede: städtisch, stadtnah, ländlich abgelegen · Stadt-Land-Vergleich · Georeferenzierung

$\overline{\text { Diplom-Pädagogin D. Müller }(\bowtie)}$

Projekt „BildungsLandschaft Oberfranken“ (BiLO), Leibniz-Institut für Bildungsverläufe e. V.

(LIfBi), Wilhelmsplatz 3, 96047 Bamberg, Deutschland

E-Mail: kontakt@lifbi.de 


\title{
Are kindergartens in remote rural regions left behind? Findings on differences in structural quality in urban-rural comparison by using the example of Upper Franconia
}

\begin{abstract}
The paper deals with the question of whether there are differences in structural quality between urban, suburban and remote rural childcare facilities. In contradiction to previous findings, this is considered detached from administrative borders and thus shows the characteristics of small regions. The results, based on data from the project "BildungsLandschaft Oberfranken (BiLO)" (Educational Landscape Upper Franconia), do not show any clear indication that, despite some differences between urban and remote rural institutions, remote rural institutions are "remote" institutions. Suburban institutions that administratively represent the rural region are at times similar to urban or remote rural institutions.
\end{abstract}

Keywords Kindergarten quality - Structural characteristics of kindergartens · Regional differences: urban, suburban, remote rural · Urban-rural comparison · Georeferencing

\section{Einleitung}

„Ob ein Kind eine gute oder schlechte Kita besucht, hängt nicht nur davon ab, in welchem Bundesland es betreut wird, sondern sogar in welchem Kreis. Es ist also der Wohnort, der innerhalb Deutschlands über die Bildungschancen von Kindern entscheidet.“ heißt es in einer Meldung der Bertelsmann Stiftung zu Ergebnissen des Ländermonitoring Frühkindliche Bildungssysteme (Stein und Bock-Famulla 2017). Bisherige Forschungsergebnisse zeigen bei einem regionalen Vergleich von strukturellen Merkmalen von Kindertageseinrichtungen wie Öffnungszeiten und Personalschlüssel länder- und kreisspezifische Unterschiede (z. B. Autorengruppe Bildungsberichterstattung 2018; Bock-Famulla et al. 2019; Hüsken 2011; Tietze 1998).

Das Kinder- und Jugendhilferecht sowie die Ausführungsgesetze der Länder zu Rahmenbedingungen und Strukturen von Kindertagesbetreuung regeln nur in Grundzügen die Vorgaben zur Qualität; einheitliche Vorgaben existieren nicht. Die Umsetzung und Sicherung struktureller Qualitätsstandards liegt damit in der Verantwortung der jeweiligen Träger der Kindertageseinrichtungen und ist vor allem im Zuge des Ausbaus des Platzangebots und damit einhergehender finanzieller Beanspruchung ein aktuell viel diskutiertes Thema.

Studien verwenden dabei in der Regel als regionale Vergleichsebene die Kreisebene oder die Ebene der Jugendamtsbezirke, sodass die Ergebnisse auf administrative Grenzen beschränkt bleiben. Sowohl die Vergabe als auch die Suche nach Betreuungsplätzen findet jedoch vorwiegend lokal statt, orientiert sich nicht an administrativen Grenzen und ist auf einen deutlich kleinräumigeren Bereich als die Kreisebene ausgerichtet (Fuchs-Rechlin 2014). Insgesamt liegen jedoch kaum Studien vor, die ausführlich die strukturellen Merkmale des Angebots auf Stadt-LandEbene oder auf der kommunalen und interkommunalen Ebene vergleichen. Wenn diese Vergleichsebene herangezogen wird, dann in der Regel, um Unterschiede in 
der Nachfrage in Form der Betreuungsquote (z. B. Hüsken 2011) zu analysieren, bei der Untersuchung der Zusammensetzung der Einrichtung und deren Auswirkungen (z. B. Lehrl et al. 2014; Leu 2007) sowie, vor allem im Zuge des Ausbaus von Kindertagesbetreuung, bei der Betrachtung der vorhandenen Anzahl an Einrichtungen und Plätzen (z. B. Bock-Famulla et al. 2019).

Daher beschäftigt sich der vorliegende Beitrag mit der Frage, ob zwischen städtischen und ländlichen Kindertageseinrichtungen Unterschiede in der pädagogischen Qualität, spezifisch in der Strukturqualität, bestehen (vgl. Abschn. 2). Mittels Bestimmung der geographischen Lage der Einrichtung wird eine Bindung an administrative Grenzen überwunden. Im Fokus der Analyse steht ein Vergleich von Einrichtungen aus städtischen und ländlich abgelegen Regionen sowie Einrichtungen aus dem städtischen Umland. Ländlich abgelegene Regionen sind häufig strukturell benachteiligt. Zum einen, weil sie nicht von der vorhandenen Infrastruktur ${ }^{1}$ der städtischen Regionen profitieren können und zum anderen, bedingt durch die Auswirkungen des sozio-demografischen Wandels, vorhandene Infrastruktur abgebaut wird, aber auch Problematiken durch eine Veränderung der Bevölkerungsstruktur entstehen wie eine alternde Bevölkerung, Abwanderung der jungen Bevölkerung und dadurch resultierender Geburtenrückgang (Dehne 2013; Munzinger 2014). Diese Faktoren könnten sich negativ auf die strukturellen Merkmale der Kindertageseinrichtung auswirken wie beispielsweise weniger qualifiziertes pädagogisches Personal sowie allgemeine Personalknappheit und damit ein höherer Personalschlüssel oder kürzere Öffnungszeiten. Ob ländlich abgelegene Einrichtungen nicht nur geographisch als ,abgelegen“ zu bezeichnen sind, sondern auch hinsichtlich der Strukturqualität der Kindertageseinrichtungen, wird nachfolgend explorativ nachgegangen. Um die Spezifika der ländlich abgelegenen Regionen deutlicher hervorzuheben, werden vergleichend nicht nur Einrichtungen aus städtischen Regionen untersucht, sondern ebenfalls Einrichtungen des städtischen Umlandes, die eine gegensätzliche Landregion darstellen. Anders als die ländlich abgelegenen Regionen profitieren die Regionen des städtischen Umlandes vom breiten Infrastrukturangebot der städtischen Region und leiden nicht unter Problematiken des sozio-demografischen Wandels. Insgesamt ermöglicht dieser spezifische Blick auf die verschiedenen Regionen ggf. heterogene Qualitätsbedarfe zu erfassen und bietet eine Grundlage für politische Entscheidungen im Qualitätsdiskurs.

Der Forschungsfrage wird am Beispiel Oberfrankens nachgegangen (vgl. Abb. 1). Die Verwaltungsregion im Norden von Bayern besitzt insgesamt eine eher ländliche Gesamtstruktur, weist keine großen Ballungsräume auf und bietet daher die Möglichkeit ländlich abgelegene Regionen zu untersuchen. Circa eine Million Einwohner verteilen sich auf vier kreisfreie Städte und neun Landkreise. Die größte Stadt ist Bamberg mit fast 80.000 Einwohnern. In Bezug zur Bevölkerungsentwicklung ist eine Zweiteilung zu erkennen. Die Region Oberfranken-Ost ${ }^{2}$ ist mit Ausnahme der kreisfreien Stadt Bayreuth von einer Bevölkerungsabnahme betrof-

\footnotetext{
${ }^{1}$ Hier ist vor allem die soziale Infrastruktur zu nennen wie beispielsweise Schulen, Kindergärten, ärztliche Versorgung, Sport- und Freizeitmöglichkeiten, Einkaufsstätten sowie kulturelle Einrichtungen.

2 Dazu zählen die Landkreise Bayreuth, Hof, Kulmbach und Wunsiedel i.F. sowie die kreisfreien Städte Bayreuth und Hof.
} 


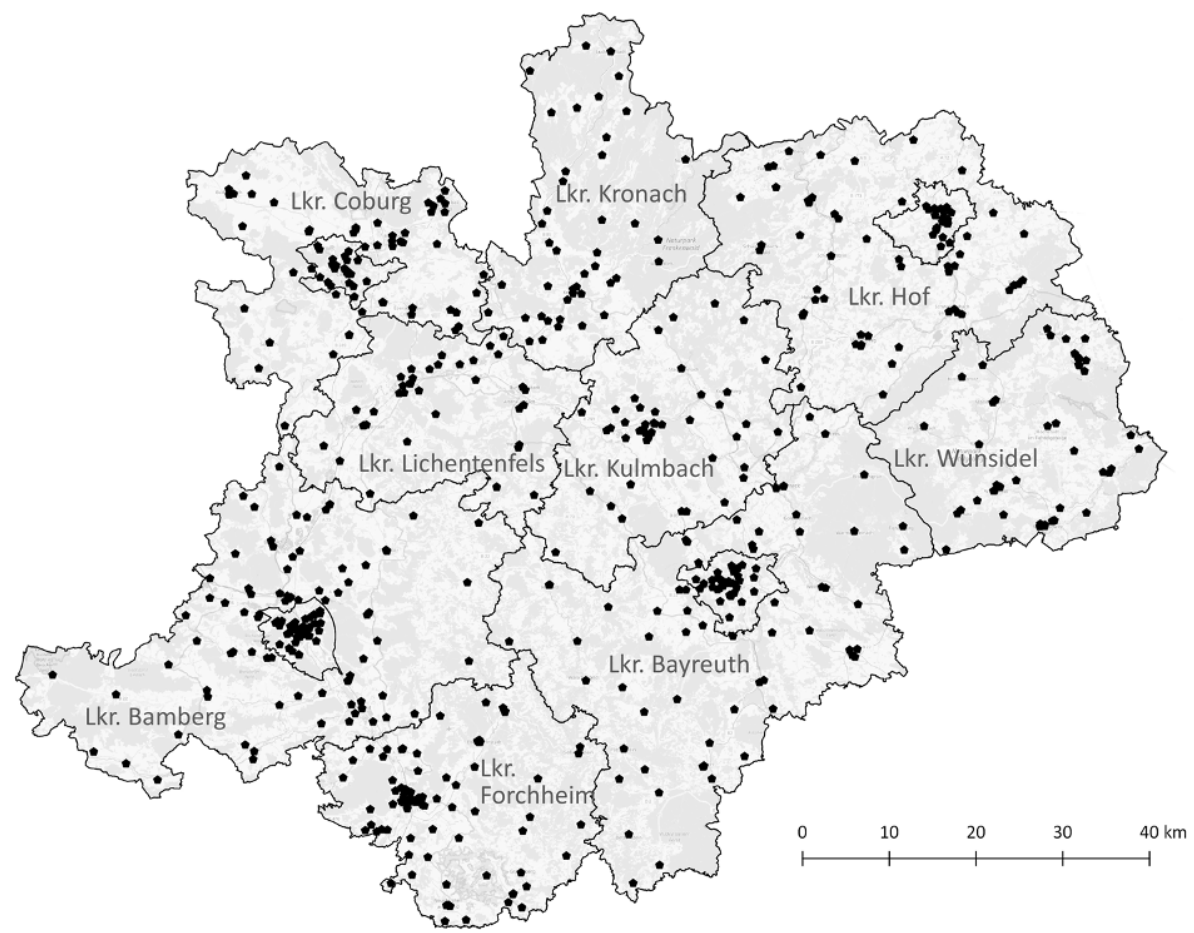

Abb. 1 Verteilung der Grundgesamtheit von Kindertageseinrichtungen in Oberfranken. (Quelle: Eigene Darstellung)

fen (1,3\% seit 2011, 15,6\% seit 1950; Stand: 31.12.2018), während die Region Oberfranken-West ${ }^{3}$ einen Bevölkerungszuwachs verzeichnen kann (1,6\% seit 2011, 12,2\% seit 1950; Stand: 31.12.2018). Dieser Zuwachs betrifft die Landkreise Bamberg, Coburg und Forchheim, während die kreisfreien Städte Bamberg und Coburg sowie die Landkreise Kronach und Lichtenfels eher gleichbleibendes Niveau bzw. eine Bevölkerungsabnahme zeigen (vgl. Bayerisches Landesamt für Statistik und Datenverarbeitung, Stand: 31.12.2018). Die Bevölkerungsvorausberechnung für das Jahr 2037 prognostiziert für beide Regionen in Oberfranken einen Bevölkerungsverlust, wobei Oberfranken-Ost mit einer Prognose von 9,1\% stärker betroffen ist als Oberfranken-West mit 2,8\%. ${ }^{4}$ In Bezug zur Altersstruktur besitzt Oberfranken im Vergleich zu den anderen Regierungsbezirken in Bayern einen höheren Anteil an älterer Bevölkerung. 46,6\% der Gesamtbevölkerung Oberfrankens ist 50 Jahre und älter, während Bayern einen Anteil von $42,9 \%$ aufweist. $\mathrm{Zu}$ erkennen ist ebenfalls ein Unterschied zwischen den beiden Regionen Oberfrankens: OberfrankenOst weist einen höheren Anteil an älteren Menschen ab 50 Jahre auf $(48,1 \%$ der Bevölkerung, davon ist die Hälfte 65 Jahre und älter) im Vergleich zu Oberfranken-

\footnotetext{
3 Dazu zählen die Landkreise Bamberg, Coburg, Forchheim, Kronach und Lichtenfels sowie die kreisfreien Städte Bamberg und Coburg.

4 Für Gesamtbayern ist hingegen ein Bevölkerungszuwachs von 3,7\% bis zum Jahr 2037 prognostiziert.
} 
West (45,4\% der Bevölkerung). Die Prognose für das Durchschnittsalter der Bevölkerung in 2037 ist in beiden Regionen Oberfrankens steigend und liegt über dem prognostizierten Wert für Bayern.

\section{Die Bedeutung der Strukturqualität für die pädagogische Qualität von Kindertageseinrichtungen}

Vor allem bei qualitativhochwertiger Betreuung besteht weitgehend Konsens in der Forschung, dass die Kindertagesbetreuung Kinder fördert (Anders et al. 2012; Becker 2010; Roßbach 2005; Vandell und Wolfe 2000). Vor allem sozial benachteiligte Kinder profitieren von einer Kindertagesbetreuung mit guter Qualität (Anders 2013; Beckh et al. 2014; Burchinal et al. 2010; Roßbach et al. 2009; Watamura et al. 2011). Es ist also davon auszugehen, dass der Besuch eines Kindergartens positive Auswirkungen auf die kognitive und soziale Kompetenzentwicklung von Kindern haben kann, wobei das Niveau und die Dauerhaftigkeit des positiven Effekts stark von der Qualität der Institution abhängig zu sein scheinen.

Eine gute pädagogische Qualität von Kindertageseinrichtungen liegt nach Tietze (2008, S. 17) vor, [...] ,,wenn die jeweiligen pädagogischen Orientierungen, Strukturen und Prozesse auf das körperliche, emotionale, soziale und intellektuelle Wohlbefinden und die Entwicklung und Bildung der Kinder in diesen Bereichen aktuell wie auch auf Zukunft gerichtet fördern und die Familien in ihrer Betreuungs- und Erziehungsaufgabe unterstützen“. Das Konzept der pädagogischen Qualität besteht nach dieser Definition aus drei Qualitätsdimensionen: Prozessqualität, Orientierungsqualität und Strukturqualität (Tietze 1998).

Die Prozessqualität umfasst Bildungsprozesse, die sich auf die Interaktion zwischen Erzieher und Kind, zwischen den Kindern selbst sowie auf die Interaktion des Kindes mit der räumlich-materiellen Umwelt beziehen. Dazu zählen beispielsweise professionelles Agieren, Reagieren, reflexives Handeln sowie die aktive Gestaltung von Anregungen und Aktivitäten zur Unterstützung der kindlichen Entwicklung und Bildung (Viernickel 2008). Durch die gezielte Stimulation der Bildung und Entwicklung des Kindes übt die Prozessqualität einen direkten Einfluss auf das Kind aus. Eine hohe Prozessqualität wirkt dabei positiv auf den Entwicklungsstand der Kinder ein (NICHD 2002, 2006; Tietze 1998).

Die Orientierungsqualität umfasst Orientierungen und Überzeugungen des pädagogischen Personals, die den Bildungsprozess beeinflussen. Dazu zählen beispielsweise Vorstellungen und Einstellungen über Erziehung und Bildung, Werte des pädagogischen Fachpersonals sowie ihr Bild vom Kind (vgl. ECCE-Study Group 1997, 1999; Tietze 1998).

Die Strukturqualität wird definiert als zeitlich relativ stabile, durch politische Entscheidungen meist veränderbare räumlich-materielle und personelle Rahmenbedingungen, unter denen die pädagogische Arbeit stattfindet (Tietze 1998; Tietze und

\footnotetext{
5 European Child Care and Education (ECCE) Study Group (1997). Cross national analyses of the quality and effects of early childhood programmes on children's development. Final report. (Unveröffentlichtes Dokument).
} 
Viernickel 2016). Dazu zählen räumlich-materielle Bedingungen wie die zur Verfügung stehenden Räume und die Anzahl verschiedener Materialien, aber auch soziale Bedingungen wie Gruppengröße und personelle Bedingungen wie Erzieherqualifikation und Personalschlüssel.

Auch wenn die Prozessqualität einen direkten Einfluss auf die kindliche Entwicklung ausübt, sind ebenso die Struktur- und Orientierungsqualität, die in Wechselwirkung zueinander stehen, durch den Einfluss auf die Prozessqualität bedeutsame Qualitätsdimensionen (Anders 2013; Petrogiannis und Melhuish 1996; Tietze 1998; Tietze et al. 2013a; Vermeer et al. 2016). Strukturelle Bedingungen bilden die Grundlage für interaktive Prozesse im pädagogischen Alltag (NICHD 2002). Einige Studien können zeigen, dass bis zu 50\% der Varianz in der Prozessqualität der Kindertageseinrichtung durch strukturelle Merkmale erklärt werden kann (BeckerStoll und Wertfein 2013; Perren et al. 2016; Pianta et al. 2005; Tietze und Lee 2009; Tietze et al. 2013a, 2005; Viernickel 2008). Vor allem der Erzieher-Kind-Schlüssel, die Gruppengröße sowie die Qualifikation der pädagogischen Fachkräfte weisen in verschiedenen Studien einen stabilen Zusammenhang in Bezug zur Prozessqualität auf (NICHD 2002; Pianta et al. 2005; Viernickel 2008; Roßbach 2005) und werden auch als „eisernes Dreieck“ der Strukturmerkmale bezeichnet (Hayes et al. 1990; Viernickel et al. 2013). Alle drei Merkmale haben einen wichtigen Einfluss auf die Qualität der Angebote vor allem in Bezug auf eine bessere Interaktionsqualität sowie zur Bereitstellung einer lernförderlichen Umgebung (Blossfeld et al. 2012; NICHD 2002; Tietze et al. 2013b, 2005; Viernickel 2008; Viernickel et al. 2013). Durch die Verantwortung für die Umsetzung der Qualitätsentwicklung und -sicherung der Einrichtung und der damit verbundenen Aufgaben besitzen neben den Trägern die Leitungskräfte der Einrichtungen eine zentrale Bedeutung für die pädagogische Qualität der Einrichtung (Strehmel 2015, S. 150 ff.; Strehmel und Ulber 2014, S. 10 ff.; Viernickel et al. 2013). Daher sind die zeitlichen Ressourcen, die Leitungskräfte für diese Aufgaben vom erzieherischen Alltag freigestellt werden, bedeutend. Studien zeigen, dass insbesondere in Einrichtungen mit wenigen Kindern, kaum Zeit für Leitungsaufgaben eingeräumt wird (Lange 2017). Nicht nur die sozialen und personellen Rahmenbedingungen haben einen Einfluss auf die Prozessqualität, sondern auch die räumlich-materiellen Rahmenbedingungen. So zeigten beispielsweise Tietze et al. (2013b) mit der NUBBEK-Studie positive Zusammenhänge mit der dem Kind zur Verfügung stehenden Fläche sowohl im Innen- als auch im Außenbereich.

Insgesamt bildet die Strukturqualität eine wichtige Stellschraube im Rahmen der Qualitätsentwicklung in den Kindertageseinrichtungen, auch wenn Verbesserungen auf der Ebene der strukturellen Rahmenbedingungen nicht alleinig ausreichend sind, um eine gute Betreuungsqualität zu sichern. Dennoch können unzureichende strukturelle Rahmenbedingungen in Kindertageseinrichtungen Kinder daran hindern, wichtige fördernde Erfahrungen zu machen (Anders 2013; NICHD 2002; Tietze 1998; Viernickel 2008). Nicht zuletzt zeigt sich, dass eine gute Strukturqualität die Zufriedenheit und das Engagement pädagogischer Fachkräfte steigert (Jungbauer und Ehlen 2015; Schreyer 2014), was sich wiederum positiv auf die kindliche Entwicklung auswirkt.

Die gesetzlichen Regelungen zu den strukturellen Merkmalen von Kindertageseinrichtungen unterscheiden sich durch die Länderhoheit zwischen den Bundeslän- 
dern. Das bundesrechtliche SGB VIII enthält keine konkreten Vorgaben, sondern beinhaltet allgemeine Regelungen zur Personalausstattung und Qualifizierung des pädagogischen Personals zur Wahrung des Kindeswohls. Die Auslegung und Überprüfung obliegt dem jeweiligen überörtlichen Träger der Jugendhilfe, in der Regel dem Landesjugendamt. Dazu kommen die gesetzlichen Regelungen der einzelnen Bundesländer, die sich in Bezug zu den strukturellen Merkmalen der Kindertageseinrichtung hinsichtlich des Verbindlichkeitsgrads, der Regelungsinhalte und -dichte unterscheiden. Es existieren keine einheitlichen Vorgaben zu strukturellen Merkmalen der Einrichtung wie Gruppengröße, zur Verfügung stehende Flächen im Außen- und Innenbereich, Qualifikation des Personals sowie Öffnungszeiten. Auch das Bayerische Kinderbildungs- und -betreuungsgesetz (BayKiBiG) sowie die dazugehörige Verordnung zur Ausführung des Bayerischen Kinderbildungs- und -betreuungsgesetzes (AVBayKiBiG) enthalten nur begrenzt gesetzliche Vorgaben zu strukturellen Merkmalen. Die gesetzlichen Grundlagen in Bayern weisen allgemeine Vorgaben zu den erforderlichen Qualifikationen des pädagogischen Personals sowie Mindestanforderungen zum Anstellungsschlüssel auf. Gruppengrößen sowie bauliche und/oder räumliche Mindeststandards sind weder im BayKiBiG noch in der AVBayKiBiG geregelt. Die Umsetzung obliegt daher den Trägern der Kindertageseinrichtungen, sodass Unterschiede zwischen den Einrichtungen auch im Bereich der strukturellen Merkmale erwartbar sind. Zudem beinhalten die gesetzlichen Regelungen nur Mindestanforderungen, die nicht mit pädagogischen Standards guter Qualität gleichzusetzen sind. Ob Unterschiede in der Strukturqualität zwischen Stadt- und Landregionen vorliegen wird nachfolgend nachgegangen.

\section{Datengrundlage}

Grundlage für die Analyse bilden Daten aus der Befragung von Kindertageseinrichtungen aus dem Projekt „BildungsLandschaft Oberfranken (BiLO)“, das von der Oberfrankenstiftung finanziert und am Leibniz-Institut für Bildungsverläufe e.V. (LIfBi) angesiedelt ist (vgl. Sixt et al. 2017).

Die Datengrundlage bilden Befragungsdaten aus einer Vollerhebung aller Kindertageseinrichtungen ${ }^{6}$ in Oberfranken im Januar bis März 2016. Da keine vollständige Liste aller Einrichtungen existiert, wurden die von den öffentlichen Trägern der Einrichtungen zur Verfügung gestellten Anschriftenverzeichnisse um eine eigene Internetrecherche nach Einrichtungen ergänzt (Stand: Herbst 2015). Es konnten 607 Kindertageseinrichtungen ${ }^{7}$ ermittelt werden, darunter 41 Krippen, 134

\footnotetext{
${ }^{6}$ Kindertageseinrichtungen sind definiert als außerschulische Einrichtungen, in denen Kinder ganztägig oder für einen Teil des Tages aufgenommen sowie pflegerisch und erzieherisch regelmäßig betreut werden, wie z. B. Kinderkrippen, Kindergärten und Häuser für Kinder (BayKiBiG Art. 2 Abs. 1) sowie sonstige Einrichtungen, die die Kriterien des BayKiBiG erfüllen und über eine Betriebserlaubnis nach $\S 45$ SGB VIII verfügen (z.B. auch Modellprojekt Netz für Kinder und Elterninitiativen). Ausgenommen sind Horte und Einrichtungen in Wirtschaftsunternehmen (sogenannte Betriebskindergärten). Ebenfalls unberücksichtigt bleibt der Bereich der Tagespflege.

7 Die Statistik weist 665 Kindertageseinrichtungen auf (Bayerisches Landesamt für Statistik, 2015, Stichtag 1. März 2015). Die Differenz von 58 Einrichtungen verteilt sich auf 17 Krippen, 4 Kindergärten und
} 


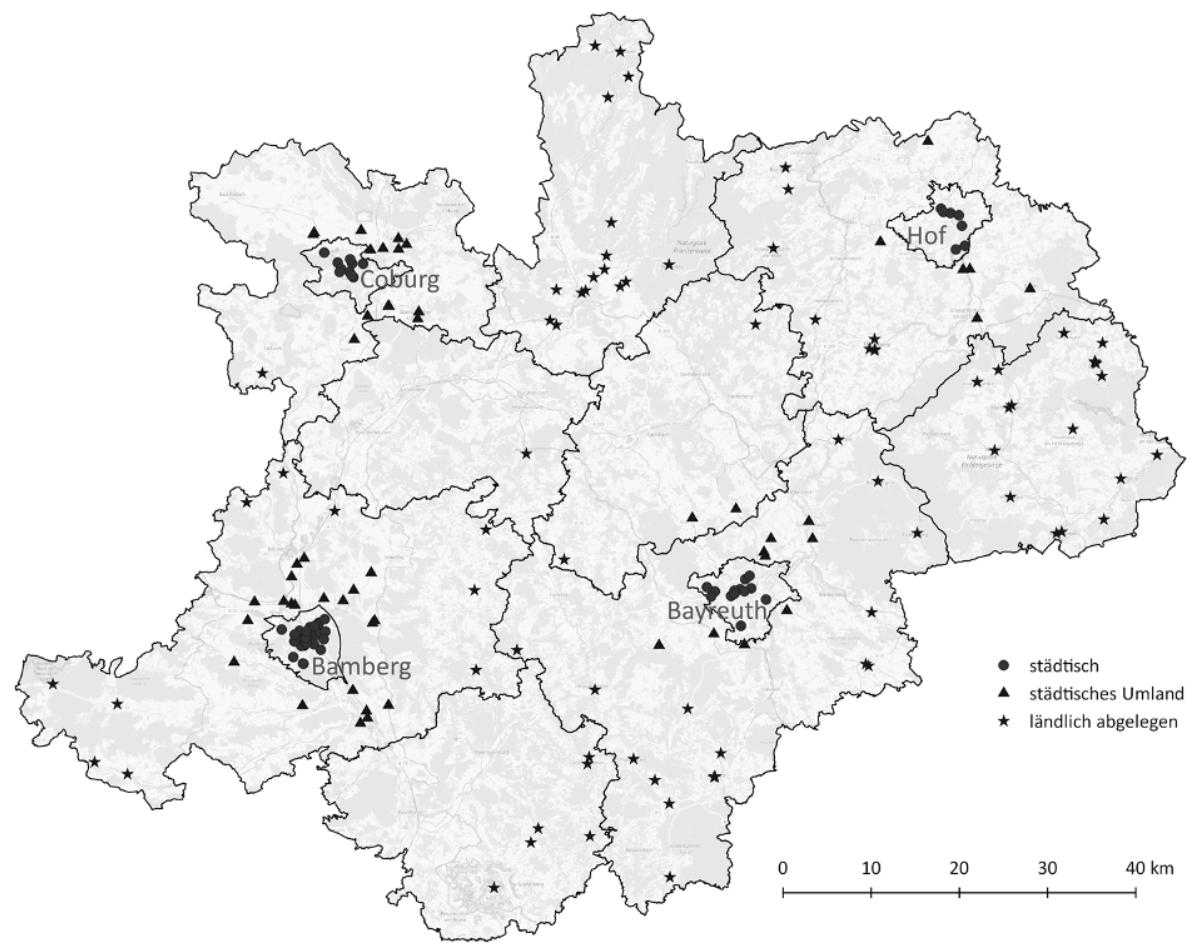

Abb. 2 Geografische Verteilung der drei Einrichtungsgruppen in Oberfranken. (Quelle: Eigene Darstellung)

Kindergärten für Kinder ab einem Alter von drei Jahren und 432 Kindertageseinrichtungen für Kinder allen Alters bis zum Schuleintritt (vgl. Abb. 1). Über einen Onlinefragbogen wurden die Einrichtungsleitungen zu strukturellen Merkmalen ihrer Einrichtung befragt. Die Teilnahmequote betrug 50\% ( $n=300)$ (vgl. Sixt et al. 2018).

Zur Einteilung in Kontrastregionen wurden mittels einer Klassifikation über die regionale Lage der Einrichtungen drei Gruppen gebildet (vgl. Abb. 2). ${ }^{8}$ Dazu wurden die Adressen der Einrichtungen georeferenziert und die Distanzen zu den umliegenden Städten, zur Zuordnung in die jeweilige Substichprobe, auf Basis des Straßenverlaufs berechnet (vgl. Hofmann 2018). Als Einrichtungen in städtischer Lage wurden jene klassifiziert, die in Städten mit über 40.000 Einwohnern liegen.

37 altersübergreifende Einrichtungen. Ein Teil der Differenz kann neben Schließungen von Einrichtungen über die Aufnahme von Spielkreisen und Spielgruppen in der Statistik erklärt werden, die der Definition von Kindertageseinrichtungen entsprechen, jedoch durch eine Internetrecherche nur schwer zu identifizieren sind. Bedingt durch den nicht vorhandenen Zugang zu vollständigen Adresslisten von Kindertageseinrichtungen kann jedoch keine eindeutige Ursache benannt werden. Dies zeigt die Bedeutung dieses Zugangs im Bereich der frühkindlichen Bildung.

8 Bereits existierende Klassifikationen wie beispielsweise der Stadt- und Gemeindetyp des Bundesinstituts für Bau-, Stadt- und Raumforschung (BBSR) konnte nicht herangezogen werden, da diese im Bereich der Landregionen zu wenig differenziert ist, da ihr Fokus auf den Stadtregionen liegt und sich somit für die ländlich geprägte Struktur Oberfrankens nur zwei Vergleichsregionen ergeben hätten. 
Tab. 1 Operationalisierung der untersuchten Merkmale der Strukturqualität

\begin{tabular}{|c|c|c|}
\hline Dimension & Merkmal & Operationalisierung \\
\hline \multirow{2}{*}{$\begin{array}{l}\text { Soziale } \\
\text { Rahmen- } \\
\text { bedin- } \\
\text { gungen }\end{array}$} & $\begin{array}{l}\text { Anzahl der Kinder } \\
\text { pro Einrichtung }\end{array}$ & $\begin{array}{l}\text { Anzahl der Kinder je Einrichtung unabhängig von ihrer Besuchs- } \\
\text { dauer }\end{array}$ \\
\hline & Anzahl Warteplätze & $\begin{array}{l}\text { Anzahl der Kinder auf einer Warteliste je Einrichtung differen- } \\
\text { ziert nach Alter. Einrichtungen, die keine Warteliste haben, sind } \\
\text { mit der Anzahl } 0 \text { in die Analyse eingegangen }\end{array}$ \\
\hline
\end{tabular}

Anzahl freie Plätze

Anzahl der freien Plätze je Einrichtung differenziert nach Alter,

Personelle Anteil der pädagounabhängig ob der Platz ein Halbtages- oder Ganztagesplatz ist

Rahmen- gischen Fachkräfte Anteil der pädagogischen Fachkräfte mit akademischer Ausbilbedin- mit akademischer gungen Ausbildung (in Prozent) dung als höchsten beruflichen Bildungsabschluss (Bachelor-Kindheitspädagogik, -Pädagogik oder vergleichbarer Abschluss; Dipl.Sozialpädagogik, Dipl.-Sozialarbeiter, Dipl.-Pädagogik, Dipl.Erziehungswissenschaft (Uni, FH oder vergleichbarer Abschluss sowie entsprechender Master-Abschluss); Dipl.-Heilpädagogik) vom gesamten pädagogischen Personal der Einrichtung

Anteil der erzieheAnteil der Personen vom gesamten erzieherisch tätigen Personal risch tätigen Personen mit max. Kinderpflegerinnenbzw. Kinderpflegerausbildung (in Prozent) Erzieher-KindRelation der Einrichtung mit Kinderpflegerinnen- bzw. Kinderpflegerausbildung als höchsten beruflichen Bildungsabschluss, Praktikantinnen oder Praktikanten im Anerkennungsjahr und Personen ohne abgeschlossene Ausbildung

Relation zwischen der Anzahl der erzieherisch tätigen Personen und der Anzahl der angemeldeten Kinder pro Einrichtung. Das Alter der Kinder und die Betreuungsdauer blieben hierbei unberücksichtigt

Anteil erzieherisch Anteil der Personen vom gesamten erzieherisch tätigen Personal tätige Personen $a b$ 55 Jahre (in Prozent) der Einrichtung mit einem Alter über 55 Jahre

Leitungsfreistellung

Angabe zur Übernahme der Leitungsaufgaben je Einrichtung; 4-stufig: $1=$ keiner, $2=$ eine Person anteilig, $3=$ eine Person in Vollzeit, $4=$ mindestens zwei Personen anteilig

Räumlich- Einrichtungsgröße Die verfügbaren Quadratmeter pro angemeldetes Kind je Innenmaterielund Außenfläche je Einrichtung

le Zufriedenheit bauli-

Rahmen- cher Zustand

Die Angabe der Einrichtungsleitung zum baulichen Zustand der

bedinEinrichtung; 4-stufig: 1 = gar nicht zufrieden, $2=$ eher nicht zufriegungen

Öffnungszeiten

Elternbeitrag (in Euro) den, $3=$ eher zufrieden, $4=$ sehr zufrieden

Die Öffnungsdauer in Minuten pro Tag

Der monatliche Beitrag der Eltern für einen Platz in der Einrichtung differenziert nach Alter des Kindes

Kosten Mittagessen (in Euro) Die durchschnittlichen Kosten für ein Mittagessen pro Tag je Einrichtung

Das sind für den Regierungsbezirk Oberfranken die vier kreisfreien Städte Bamberg, Bayreuth, Coburg und Hof $(n=58)$. Ländlich abgelegene Einrichtungen $(n=80)$ sind jene, die sich in einer Distanz von mindestens 20 Kilometern zur nächsten (Mittel-) 
Stadt (ab 20.000 Einwohner) befinden..$^{9} 79 \%$ davon sind in Gebieten verortet, die von Bevölkerungsabnahme und -alterung betroffen sind..$^{10}$ Einrichtungen im städtischen Umland $(n=52)$ befinden sich in einem Radius von maximal 15 Kilometern um die vier kreisfreien Städte. Einrichtungen, die sich anhand der Klassifikation nicht einordnen ließen, sind nicht Bestandteil der Analyse. ${ }^{11}$

Die Auswahl der in die Analyse eingegangen Variablen zur Strukturqualität orientiert sich zum einen am Forschungsstand (vgl. Abschn. 2) und zum anderen an denen im Bildungsbericht angegebenen Merkmalen. Da die BiLO-Studie nur Informationen auf Einrichtungsebene erfasst hat, können keine Merkmale auf Gruppenebene berücksichtigt werden. ${ }^{12}$ Dennoch wird das „eiserne Dreieck“, das die für den Einfluss auf die Prozessqualität relevantesten Strukturmerkmale beinhaltet, mit den untersuchten Merkmalen der Erzieher-Kind-Relation, der Einrichtungsgröße und der Qualifikation der pädagogischen Fachkräfte abgebildet. Die Operationalisierung der untersuchten Merkmale kann der Tab. 1 entnommen werden.

Für den Vergleich der Mittelwerte der strukturellen Merkmale zwischen den drei Einrichtungsgruppen wurde jeweils neben einer deskriptiven Betrachtung der nichtparametrische verteilungsfreie Kruskal-Wallis-Test (Kruskal und Wallis 1952) durchgeführt, da teilweise Verletzungen in den Voraussetzungen zur Durchführung einer einseitigen Varianzanalyse vorliegen. Zur Bestimmung, welche Gruppen sich unterscheiden, fand der Bonferroni Post-Hoc-Test Verwendung, der die einzelnen Gruppen paarweise vergleicht.

\section{Ergebnisse}

Nachfolgend werden die Ergebnisse des Vergleichs der strukturellen Merkmale je Dimension der Strukturqualität dargestellt.

\subsection{Soziale Rahmenbedingungen}

In Bezug zur Anzahl der Kinder pro Einrichtung zeigt sich, dass in Einrichtungen in städtischen Regionen mehr Kinder pro Einrichtung vorhanden sind als im Vergleich zu Einrichtungen in ländlich abgelegenen Regionen (vgl. Tab. 2). Einrichtungen im

\footnotetext{
9 Der 20-Kilometer-Radius ist u. a. durch die Stichprobengröße entstanden. Bei der Wahl eines größeren Radius wäre die Stichprobe zu klein gewesen. Dennoch zeigten die Analysen mit einem größeren Radius vergleichbare Ergebnisse zu einem 20-Kilometer-Radius. Neben Kilometern wurden analog Analysen mit der Fahrtzeit (Dauer in Minuten), die man mit dem Auto benötigt, berechnet. Auch diese Analysen ergaben die gleichen Ergebnisse.

10 Davon liegen 46 Einrichtungen in der Region Oberfranken-Ost sowie 16 Einrichtungen im Landkreis Kronach und eine Einrichtung im Landkreis Lichtenfels, dir zur Region Oberfranken-West zählen.

11 Diese Einrichtungen liegen in den Landgemeinden von Oberfrankens zwischen den Einrichtungen im städtischen Umland und den ländlich abgelegenen Einrichtungen $(n=110)$ und befinden sich somit über $15 \mathrm{~km}$ Umkreis um die vier kreisfreien Städte sowie unter $20 \mathrm{~km}$ zur nächsten (Mittel-) Stadt ab 20.000 Einwohner.

12 Somit können keine Vergleiche bezüglich der Gruppengröße angestellt werden, auch wenn dieses Merkmal sich in Bezug auf die Prozessqualität als bedeutsam erwiesen hat. Ebenso kann kein Personalschlüssel berechnet werden, sondern nur eine Erzieher-Kind-Relation auf Einrichtungsebene.
} 


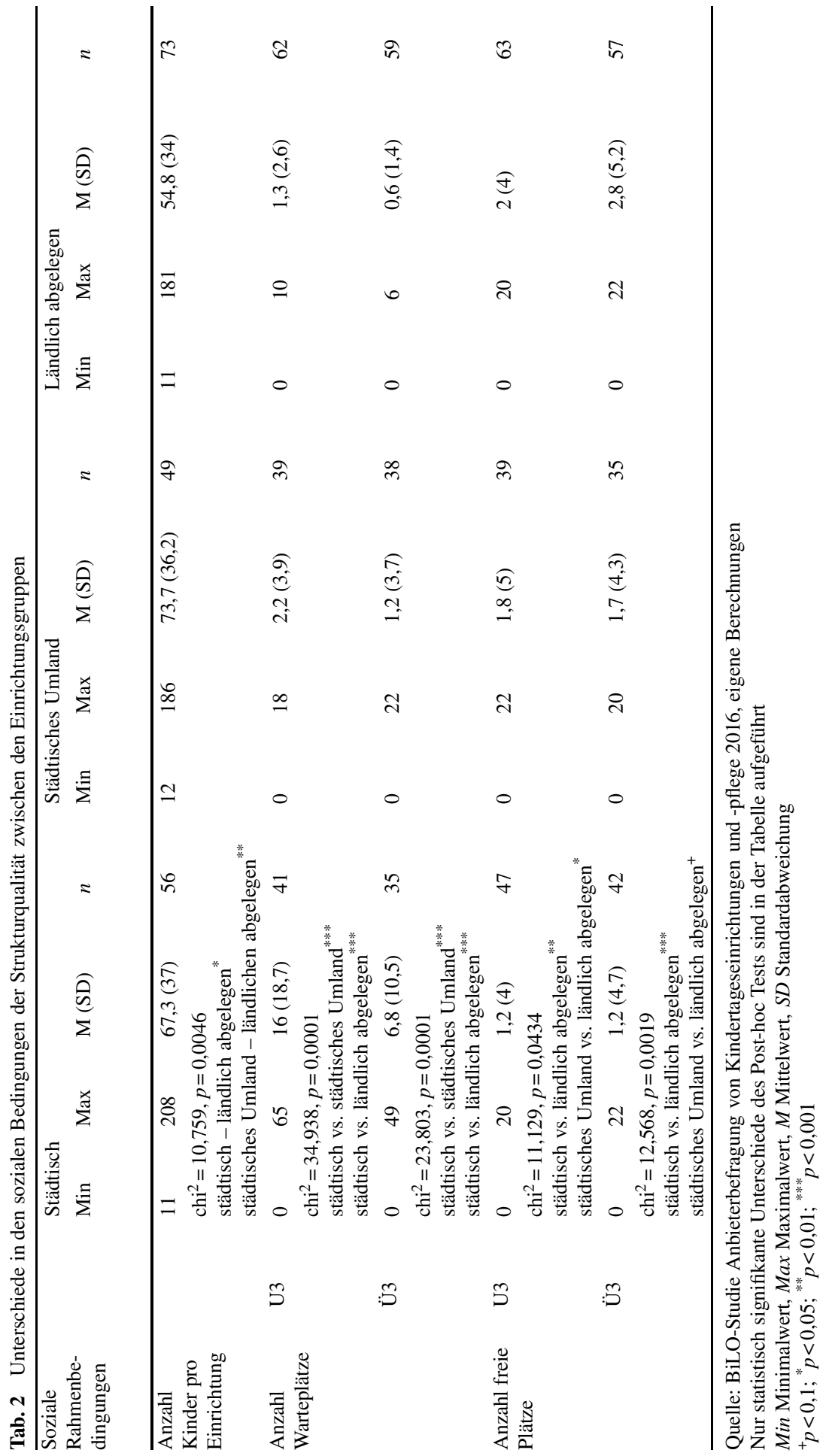


städtischen Umland sind in diesem Merkmal den städtischen Einrichtungen ähnlicher. Sie unterscheiden sich zu den Einrichtungen in den ländlich abgelegenen Regionen um durchschnittlich 19 Kinder mehr pro Einrichtung.

Hinsichtlich der Anzahl der Wartelistenplätze zeigen sich sowohl im Bereich für Kinder unter drei Jahre (U3) als auch im Bereich für Kinder über drei Jahre (Ü3) Unterschiede zu den städtischen Einrichtungen. Während in beiden ländlichen Regionen kaum Einrichtungen vorhanden sind, die Kindern keinen Platz anbieten können, ist dies in städtischen Regionen weitaus häufiger und verstärkt im U3Bereich zu finden.

Entsprechend zeigen sich Unterschiede im Vergleich der Anzahl der freien Plätze pro Einrichtung. Hervorzuheben ist hier jedoch die Situation in Einrichtungen im städtischen Umland, die anders als in ländlich abgelegenen Einrichtungen, nur in Einzelfällen Einrichtungen mit freien Plätzen aufweisen. D.h. während in städtischen Einrichtungen eher ein Platzmangel vorherrscht, ist das Platzangebot in Einrichtungen im städtischen Umland eher ausgeglichen und Einrichtungen in ländlich abgelegenen Regionen weisen durchschnittlich einen Platzüberschuss auf. Dies bedeutet auch, dass je ländlicher die Kindertageseinrichtung liegt, desto höher ist die Wahrscheinlichkeit, einen Platz zu erhalten, auch wenn die Auswahlmöglichkeiten an Einrichtungen mit der Ländlichkeit der Region abnehmen (vgl. Müller 2018).

\subsection{Personelle Rahmenbedingungen}

In Bezug zum Anteil der pädagogischen Fachkräfte mit akademischer Ausbildung zeigt sich ein deutlicher Unterschied zwischen den Einrichtungen in den beiden Landregionen im Vergleich zu den städtischen Einrichtungen. Ein statistisch signifikanter Unterschied besteht jedoch nur zwischen städtischen und ländlich abgelegenen Einrichtungen. Der Anteil an Fachkräften mit akademischem Abschluss geht in ländlich abgelegenen Einrichtungen gegen Null, während in städtischen Regionen jede dritte Einrichtung mindestens eine pädagogische Fachkraft mit akademischer Ausbildung aufweist und durchschnittlich vier Prozent des Personals einer städtischen Einrichtung akademisch qualifizierte Fachkräfte umfassen (vgl. Tab. 3). Jedoch liegen alle Einrichtungsgruppen unter dem bundesdeutschen Durchschnitt von ca. fünf Prozent (Autorengruppe Bildungsberichterstattung 2018, S. 78). Vergleicht man hingegen den Anteil an erzieherisch tätigen Personen, die als höchsten beruflichen Abschluss die Ausbildung zur Kinderpflegerin oder Kinderpfleger abgeschlossen haben, zeigen die Daten keine Unterschiede in den drei regionalen Gruppen.

Zur Deckung der Personalbedarfe bedingt durch den Ausbau der Kindertagesbetreuung ist neben einem deutschlandweiten Zuwachs des erzieherisch tätigen Personal bei den 20- bis 34-Jährigen ebenfalls ein Zuwachs bei den 50- bis 64-Jährigen zu verzeichnen, wobei sich insgesamt die Altersstruktur des pädagogisch tätigen Personen zu einem höheren Anteil an älteren Personal verschiebt (Autorengruppe Bildungsberichterstattung 2018, S. 78). In den vorliegenden Daten kann in Bezug zur Altersverteilung keine Unterschiede zwischen den Einrichtungsgruppen festgestellt werden (vgl. Tab. 3; beispielhaft der Altersbereich über 55 Jahre). Der Anteil des Personals über 55 Jahre liegt durchschnittlich bei $18 \%$. 


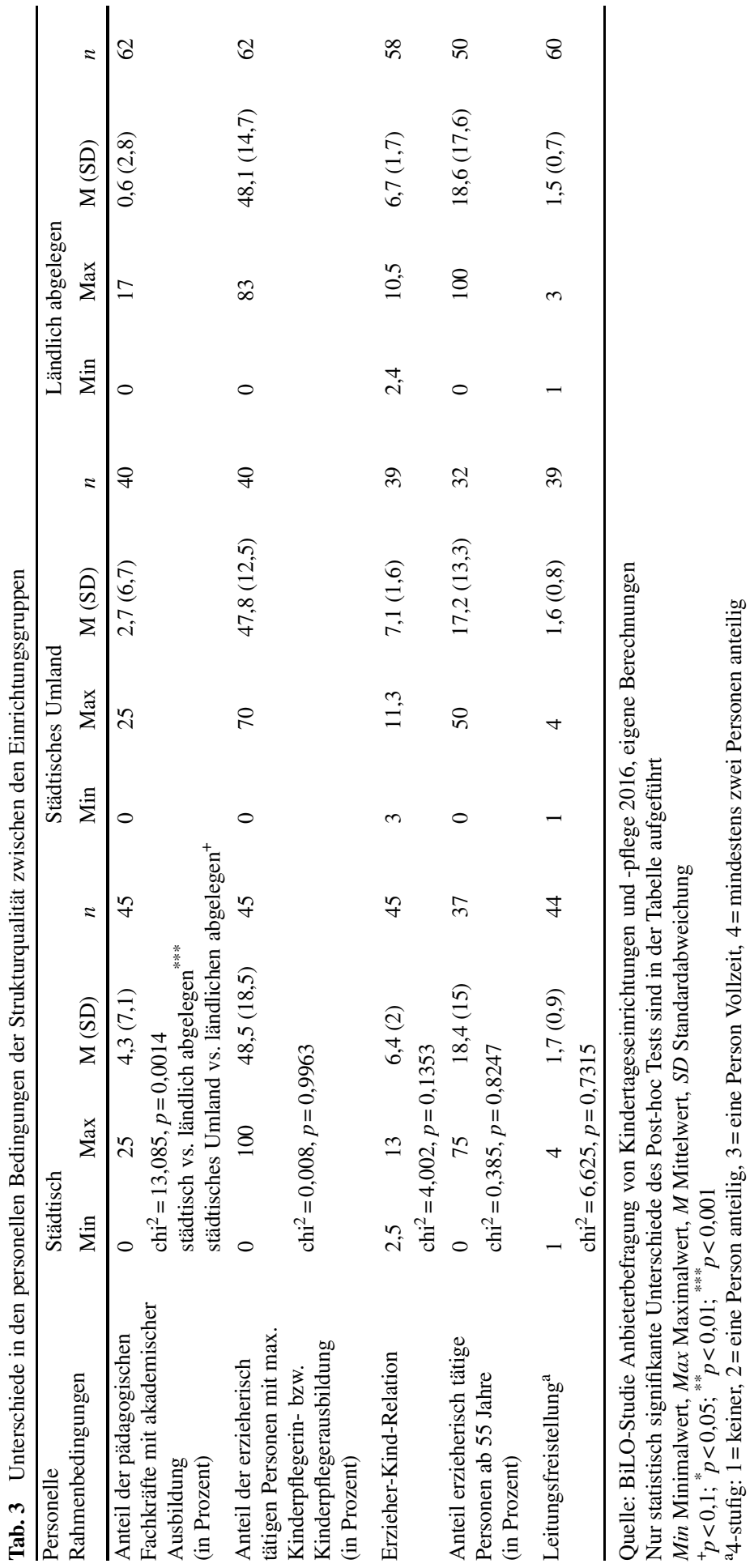


Mit Blick auf die drei Vergleichsgruppen zeigt sich auch bei der Erzieher-KindRelation, die bei durchschnittlich sieben Kindern pro erzieherisch tätige Person liegt, keine Unterschiede.

Ebenso weist das Merkmal der Leitungsfreistellung zwischen den drei Einrichtungsgruppen keine statistisch signifikanten Unterschiede auf. In Bezug zur Qualität ist jedoch insgesamt negativ festzustellen, dass in jeder zweiten Einrichtung keine Person anteilig für die anfallenden Leitungsaufgaben freigestellt ist.

\subsection{Räumlich-materielle Rahmenbedingungen}

Sowohl die Größe der Einrichtung, definiert über die verfügbaren Quadratmeter pro Kind je Innen- und Außenfläche, als auch die Zufriedenheit mit dem baulichen Zustand zeigen sich keine Unterschiede zwischen den drei Einrichtungsgruppen in den Daten (vgl. Tab. 4).

Die Öffnungszeiten sind aus der Sicht der Eltern ein wichtiges Merkmal der Qualität von Kindertageseinrichtungen, vor allem bedingt durch die zunehmende Flexibilisierung der Arbeitszeiten und die Zunahme der Frauenerwerbstätigkeit (FuchsRechlin 2011). Beim Vergleich der drei Gruppen kann in den vorliegenden Daten ein leichter Unterschied zwischen den städtischen und den ländlich abgelegenen Einrichtungen festgestellt werden. Städtische Einrichtungen haben durchschnittlich eine um 23 min $^{13}$ längere Öffnungsdauer als Einrichtungen in ländlich abgelegenen Regionen.

In Bayern sind die Träger der Einrichtung für die Festsetzung des Elternbeitrags zuständig, sodass erhebliche Unterschiede zwischen den Kommunen existieren (Autorengruppe Bildungsberichterstattung 2018, S. 70). Auch zwischen den städtischen und ländlich abgelegenen Einrichtungen zeigen sich deutliche Unterschiede im Elternbeitrag für einen Ganztagesplatz ${ }^{14}$ sowohl im U3, als auch im Ü3-Bereich. Durchschnittlich muss für einen U3-Platz in einer städtischen Einrichtung fast $60 €$ pro Monat mehr von den Eltern getragen werden als vergleichsweise in ländlich abgelegenen Einrichtungen. Der Unterschied für einen Ü3-Platz ist mit $20 €$ pro Monat deutlich geringer. D.h. je ländlicher die Kindertageseinrichtung verortet ist, desto finanziell günstiger ist ein Platz. In Bezug zu den Kosten für das in der Einrichtung angebotene Mittagsessen sind keine Unterschiede zwischen den Einrichtungen zu erkennen.

\section{Fazit}

Strukturelle Merkmale einer Kindertageseinrichtung bilden die Grundlage für pädagogische Prozesse und sind eine wichtige Stellschraube im Rahmen der Qualitätsentwicklung, auch wenn Verbesserungen auf der Ebene der strukturellen Rahmenbedingungen nicht alleinig ausreichend sind, um eine gute Betreuungsqualität zu sichern.

\footnotetext{
13 Am Beispiel des Montags.

14 Ein Ganztagesplatz entspricht fünf Tagen pro Woche mit sieben bis acht Stunden pro Tag.
} 


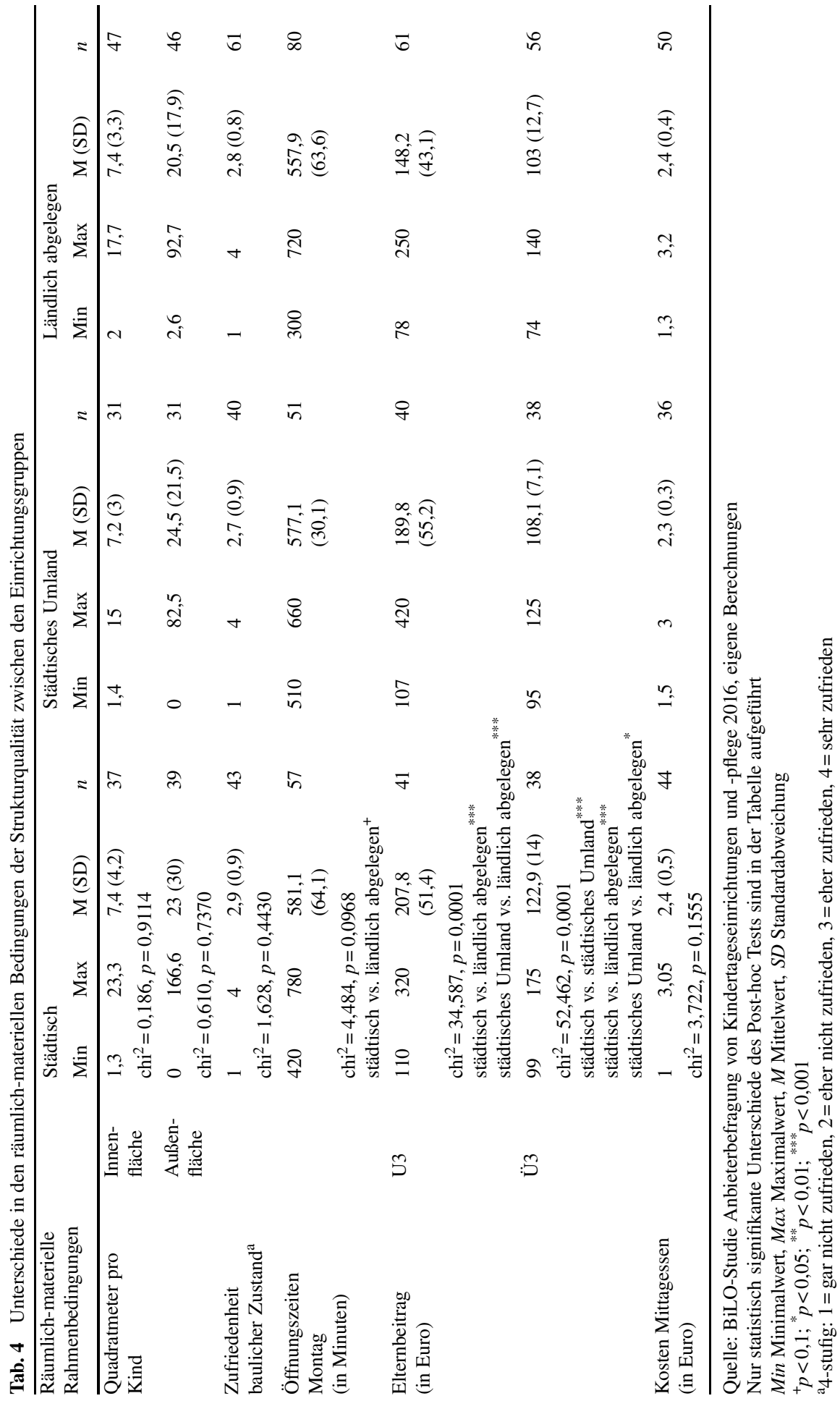


Bisherige Forschungsergebnisse konnten zeigen, dass strukturelle Merkmale von Einrichtungen zwischen Kreisen und Jugendamtsbezirken differieren und somit der Wohnort mit dazu beiträgt, Zugang zu guter pädagogischer Qualität zu erhalten. Vor diesem Hintergrund ging der Beitrag der Frage nach, ob zwischen Einrichtungen aus städtischen und ländlich abgelegen Regionen sowie Einrichtungen aus dem städtischen Umland Unterschiede in der Strukturqualität bestehen. Im Fokus dabei standen Einrichtungen aus ländlich abgelegenen Regionen, die vor allem bedingt durch den demografischen Wandel zunehmend von strukturellen Problemen des Kontextes betroffen sind. Die beiden anderen Regionen dienten dabei als Kontrastregionen.

Die Ergebnisse zeigen, dass Unterschiede in den strukturellen Merkmalen zwischen städtischen und ländlich abgelegenen Einrichtungen vorhanden sind. Ländlich abgelegene Einrichtungen besitzen kaum einen Anteil an akademisch qualifiziertem Personal, was sich negativ auf die Prozessqualität der Einrichtungen auswirken kann. Je ländlicher die Einrichtung liegt, desto geringer ist die Wahrscheinlichkeit pädagogische Fachkräfte mit akademischer Ausbildung in der Einrichtung vorzufinden. Des Weiteren ist ein Kindergartenplatz in einer ländlich abgelegenen Einrichtung für Eltern finanziell deutlich günstiger (vor allem für U3-Kinder), was aus Sicht der Eltern wahrscheinlich eher als Vorteil gewertet wird. Inwieweit die Unterschiede in den drei Einrichtungsgruppen im Elternbeitrag Auswirkungen auf die Qualität der Einrichtung hat, kann mit dem vorliegenden Beitrag nicht beantwortet werden. Für die Ursache regionsabhängiger Unterschiede im Elternbeitrag können nur Annahmen aufgestellt werden. Ein höherer Elternbeitrag kann, wenn er für Verbesserungen struktureller Merkmale eingesetzt wird wie für besondere Ausstattung und Materialien sowie für qualifiziertes Personal, positiv wirken. Ein höherer Elternbeitrag zur Deckung höherer Betriebskosten hat jedoch keinen Effekt auf Qualitätsverbesserungen. Ergänzend haben Einrichtungen in ländlich abgelegenen Regionen eine geringere Anzahl von Kindern, was jedoch bei nicht vorhandenen Unterschieden in der Personalausstattung und in der Größe der Einrichtung zwischen den drei Einrichtungsgruppen, nicht direkt als Vorteil für eine gute Strukturqualität wirken kann. In Bezug zur Quantität des Angebots ist es in ländlich abgelegenen Regionen deutlich wahrscheinlicher einen Platz für ein Kind zu erhalten. Einrichtungen mit Wartelisten sind kaum vorhanden und die Zahl freier Plätze übersteigt die Anzahl an Kindern auf Wartelisten.

Darüber hinaus zeigen sich diese Unterschiede in den oben aufgeführten strukturellen Merkmalen ebenso innerhalb der beiden Landregionen. Die Einrichtungen im städtischen Umland sind in der Regel den städtischen Einrichtungen ähnlicher. Einzige Ausnahme bildet die Anzahl an Warteplätzen in den Einrichtungen, die deutlich geringer ist als in städtischen Einrichtungen und ähnlich dem Merkmal in ländlich abgelegenen Regionen.

In Bezug zu den wichtigsten strukturellen Merkmalen des „eisernen Dreiecks“ (vgl. Abschn. 2) sticht vor allem die schlechtere Versorgung von pädagogischem Personal mit akademischer Ausbildung in ländlich abgelegenen Regionen hervor. Der einstigen Forderung des Aktionsrats Bildung (Blossfeld et al. 2012), dass zur Verbesserung und Stabilisierung von pädagogischer Qualität eine Bildung von multiprofessionellen Teams mit mindestens einer akademisch ausgebildeten Fachkraft pro Einrichtung notwendig sei, kann aktuell vor allem in ländlich abgelegenen Re- 
gionen nicht nachgekommen werden. Dies zeigt deutlich, dass innerhalb der Debatte der Professionalisierung von pädagogischen Fachkräften in Kindertageseinrichtungen ebenso regionale Herausforderung Beachtung finden müssen.

Ein einzelner Faktor bedingt jedoch keine schlechtere Strukturqualität, sodass zusammenfassend trotz Unterschieden zwischen den drei Regionen die Ergebnisse daraufhin deuten, dass unabhängig von der geografischen Lage einer Kindertageseinrichtung in Stadt- oder Landregionen gleichwertige strukturelle Bedingungen von Kindertageseinrichtungen vorzufinden sind. Kindertageseinrichtungen in ländlich abgelegenen Regionen stellen demnach in Bezug zur Strukturqualität keine „abgehängte“ Gruppe dar.

Bedingt durch die kleine Stichprobe und die speziell ländliche Region Oberfrankens, kann das Ergebnis nicht den Anspruch erheben, für alle bundesdeutschen Kindergärten repräsentativ zu sein. Dieses Ergebnis hebt dennoch die Bedeutsamkeit kleinräumiger regionaler Analysen hervor und dass je nach Fragestellung die Bindung an administrative Grenzen nicht ausreichend ist, um Spezifika von Regionen herauszuarbeiten. Eine Relevanz dieser Analysen besteht vor allem für politische Entscheidungen, da so gezielter Strategien entwickelt und Maßnahmen getroffenen werden können - beispielsweise zur Steigerung der Arbeitgeberattraktivität in Kindertageseinrichtungen in ländlichen Regionen für akademisch qualifiziertes Personal.

Danksagung Die vorliegende Arbeit ist im Rahmen des Projekts „BildungsLandschaft Oberfranken“ (BiLO) entstanden. Ich danke der Oberfrankenstiftung für die Förderung des Projekts sowie allen an der Befragung teilgenommenen Kindertageseinrichtungen. Ein weiterer Dank gilt allen an dem Projekt beteiligten Kolleginnen und Kollegen sowie Hilfskräften.

Funding Open Access funding provided by Projekt DEAL.

Open Access Dieser Artikel wird unter der Creative Commons Namensnennung 4.0 International Lizenz veröffentlicht, welche die Nutzung, Vervielfältigung, Bearbeitung, Verbreitung und Wiedergabe in jeglichem Medium und Format erlaubt, sofern Sie den/die ursprünglichen Autor(en) und die Quelle ordnungsgemäß nennen, einen Link zur Creative Commons Lizenz beifügen und angeben, ob Änderungen vorgenommen wurden.

Die in diesem Artikel enthaltenen Bilder und sonstiges Drittmaterial unterliegen ebenfalls der genannten Creative Commons Lizenz, sofern sich aus der Abbildungslegende nichts anderes ergibt. Sofern das betreffende Material nicht unter der genannten Creative Commons Lizenz steht und die betreffende Handlung nicht nach gesetzlichen Vorschriften erlaubt ist, ist für die oben aufgeführten Weiterverwendungen des Materials die Einwilligung des jeweiligen Rechteinhabers einzuholen.

Weitere Details zur Lizenz entnehmen Sie bitte der Lizenzinformation auf http://creativecommons.org/ licenses/by/4.0/deed.de.

\section{Literatur}

Anders, Y. (2013). Stichwort: Auswirkungen frühkindlicher institutioneller Betreuung und Bildung. Zeitschrift für Erziehungswissenschaft. https://doi.org/10.1007/s11618-013-0357-5.

Anders, Y., Rossbach, H.-G., Weinert, S., Ebert, S., Kuger, S., Lehrl, S., \& von Maurice, J. (2012). Home and preschool learning environments and their relations to the development of early numeracy skills. Early Childhood Research Quarterly, 27, 231-244.

Autorengruppe Bildungsberichterstattung (2018). Bildung in Deutschland 2018: Ein indikatorengestützter Bericht mit einer Analyse zu Bildung und Migration. https://www.bildungsbericht.de/de/ 
bildungsberichte-seit-2006/bildungsbericht-2018/pdf-bildungsbericht-2018/bildungsbericht-2018. pdf. Zugegriffen: 10. Okt. 2019.

Becker, B. (2010). Wer profitiert mehr vom Kindergarten? Kölner Zeitschrift für Soziologie und Sozialpsychologie, 62, 139-163.

Becker-Stoll, F., \& Wertfein, M. (2013). Qualitätsmessung und Qualitätsentwicklung in Kindertageseinrichtungen. In M. Stamm \& D. Edelmann (Hrsg.), Handbuch frühkindliche Bildungsforschung (S. 845-856). Wiesbaden: Springer VS.

Beckh, K., Mayer, D., Berkic, J., \& Becker-Stoll, F. (2014). Der Einfluss der Einrichtungsqualität auf die sprachliche und sozial-emotionale Entwicklung von Kindern mit und ohne Migrationshintergrund. Frühe Bildung, 3(2), 73-81.

Blossfeld, H.-P., Bos, W., Daniel, H.-D., Hannover, B., Lenzen, D., Prenzel, M., Roßbach, H.-G., Tippelt, R., \& Wößmann, L. (Hrsg.). (2012). Professionalisierung in der Frühpädagogik: Qualifikationsniveau und -bedingungen des Personals in Kindertagesstätten; Gutachten. Münster: Waxmann.

Bock-Famulla, K., Münchow, A., Frings, J., Kempf, F., \& Schütz, J. (2019). Länderreport Frühkindliche Bildungssysteme 2019: Transparenz schaffen - Governance stärken. Gütersloh: Bertelsmann Stiftung.

Burchinal, M., Vandergrift, N., Pianta, R., \& Mashburn, A. (2010). Threshold analysis of association between child care quality and child outcomes for low-income children in pre-kindergarten programs. Early Childhood Research Quarterly, 25, 166-176.

Dehne, P. (2013). Ein Umbau der Daseinsvorsorge in ländlichen Regionen ist notwendig. In Bundesanstalt für Landwirtschaft und Ernährung (Hrsg.), Daseinsvorsorge in ländlichen Räumen unter Druck. Wie reagieren auf den demografischen Wandel? (S. 6-8). Bonn: Bundesministerium für Arbeit und Soziales.

European Child Care and Education (ECCE) Study Group (1999). School-age assessment of child development: Long-term impact of pre-school experiences on school success and family-school relationships. http://www.uni-bamberg.de/fileadmin/uni/fakultaeten/ppp_lehrstuehle/elementarpaedagogik/Team/ Rossbach/Ecce_Study_Group.pdf. Zugegriffen: 10. Okt. 2019.

Fuchs-Rechlin, K. (2014). Der Einfluss sozioökonomischer und sozialstruktureller Bedingungen auf die Betreuungsentscheidung von Eltern. In K. Fuchs-Rechlin, G. Kaufhold, M. Thuilot \& T. Webs (Hrsg.), Der U3-Ausbau im Endspurt. Analysen zu kommunalen Betreuungsbedarfen und Betreuungswünschen von Eltern (S. 88-109). Dortmund: Eigenverlag Forschungsverbund DJI/TU Dortmund an der Fakultät 12 der Technischen Universität Dortmund.

Fuchs-Rechlin, K. (2011). Die flexible KiTa - Pluralisierte Elternwünsche, institutionelle Erfordernisse und pädagogische Grenzziehungen. KomDat Jugendhilfe, 14(1/2), 16-18.

Hayes, C. D., Palmer, J. L., \& Zaslow, M. J. (Hrsg.). (1990). Who cares for America's children. Child care policy for the 1990s. Washington: National Academy Press. By Panel on Child Care Policy, Committee on Child Development Research and Public Policy, Commission on Behavioral and Social Sciences and Education, National Research Council

Hofmann, J. (2018). Wie lassen sich räumliche Distanzen auf Straßenverlaufsebene in der Sozialforschung nutzen? LIfBi Working Paper Nr. 72. Bamberg: Leibniz-Institut für Bildungsverläufe e. V..

Hüsken, K. (2011). Kita vor Ort. Betreuungsatlas auf Ebene der Jugendamtsbezirke 2010. München: Deutsches Jugendinstitut e. V..

Jungbauer, J., \& Ehlen, S. (2015). Stressbelastungen und Burnout-Risiko bei Erzieherinnen in Kindertagesstätten: Ergebnisse einer Fragebogenstudie. Gesundheitswesen (Bundesverband der Arzte des Offentlichen Gesundheitsdienstes (Germany)). https://doi.org/10.1055/s-0034-1381995.

Kruskal, W.H., \& Wallis, W. A. (1952). Use of ranks in one-criterion variance analysis. Journal of the American Statistical Association, 47(160), 583-621.

Lange, J. (2017). Leitung von Kindertageseinrichtungen. Eine Bestandsaufnahme von Leitungskräften und Leitungsstrukturen in Deutschland. https://www.bertelsmann-stiftung.de/fileadmin/files/BSt/ Publikationen/GrauePublikationen/Leitung_von_Kindertageseinrichtungen.pdf. Zugegriffen: 1. Nov. 2019.

Lehrl, S., Kuger, S., \& Anders, Y. (2014). Soziale Disparitäten beim Zugang zu Kindergartenqualität und differenzielle Konsequenzen für die vorschulische mathematische Entwicklung. Unterrichtswissenschaft, 42(2), 132-151.

Leu, H. R. (2007). Migration im Kita-Alter: Segregation - schon im Kindergarten? DJI Bulletin, 80, $23-26$.

Müller, D. (2018). Wer besucht die nächstgelegende Kindertageseinrichtung? Analysen zum Einfluss räumlicher Faktoren bei der Einrichtungswahl. In M. Sixt, M. Bayer \& D. Müller (Hrsg.), Bildungsentscheidungen und lokales Angebot. Die Bedeutung der Infrastruktur für Bildungsentscheidungen im Lebensverlauf (S. 57-85). Münster: Waxmann. 
Munzinger, T. (2014). Demografischer Wandel: Handlungsbedarfe in den Kommunen - politische Strategien. In Bundesinstitut für Bau-, Stadt- und Raumforschung (BBSR) (Hrsg.), Vom demografischen Wandel besonders betroffene Regionen. Ein wichtiges Thema im Kontext der Demografiestrategie. Bonn: Bundesinstitut für Bau-, Stadt- und Raumforschung (BBSR).

National Institute of Child Health and Human Development Early Child Care Research Network (2002). Child-care structure-process-outcome: direct and indirect effects of child-care quality on young children's development. Psychological Science, 13, 199-206.

National Institute of Child Health and Human Development Early Child Care Research Network (2006). Child-care effect sizes for the NICHD study of EarlyCare and Youth Development. American Psychologist, 61, 99-116.

Perren, S., Frei, D., \& Herrmann, S. (2016). Pädagogische Qualität in frühkindlichen Bildungs- und Betreuungseinrichtungen in der Schweiz: Erste Erfahrungen und Befunde mit dem CLASS Toddler Beobachtungsverfahren. Konstanz: KOPS Universität Konstanz.

Petrogiannis, K., \& Melhuish, E. (1996). Aspects of quality in Greek day care centres. European Journal of Psychology of Education, 11, 177-191.

Pianta, R., Howes, C., Burchinal, M., Bryant, D., Clifford, R., Early, D., \& Barbarin, O. (2005). Features of pre-kindergarten programs, classrooms, and teachers: do they predict observed classroom quality and child-teacher interactions? Applied Developmental Science. https://doi.org/10.1207/ s1532480xads0903_2.

Roßbach, H.-G. (2005). Effekte qualitativ guter Betreuung, Bildung und Erziehung im frühen Kindesalter auf Kinder und ihre Familien. In Sachverständigenkommission 12. Kinder- und Jugendbericht (Hrsg.), Bildung, Betreuung und Erziehung von Kindern unter 6 Jahren (S. 55-174). München: Deutsches Jugendinstitut.

Roßbach, H.-G., Kluczniok, K., \& Kuger, S. (2009). Auswirkungen eines Kindergartenbesuchs auf den kognitiv-leistungsbezogenen Entwicklungsstand von Kindern. In H.-G. Roßbach \& H.-P. Blossfeld (Hrsg.), Frühpädagogische Förderung in Institutionen (S. 139-158). Wiesbaden: VS.

Schreyer, I. (2014). AQUA - Arbeitsplatz und Qualität in Kitas: Ergebnisse einer bundesweiten Befragung. München: Staatsinstitut für Frühpädagogik.

Sixt, M., Baur, H.-R., Gerbig, F., Hofmann, J., Müller, D., Stöhr, I., Thürer, S., Zeichner, C., \& Bayer, M. (2017). Das Projekt „BildunsLandschaft Oberfranken (BiLO)“ - eine Skizze. LIfBi Working Paper Nr. 71. Bamberg: Leibniz-Institut für Bildungsverläufe e. V.

Sixt, M., Gerbig, F., Zeichner, C., \& Müller, D. (2018). Methodenbericht zur BiLO-Studie Anbieterbefragung Kindertageseinrichtungen und -pflege 2016 in Oberfranken (Studiennummer C01) im Rahmen des Projekts „BildungsLandschaft Oberfranken (BiLO)“. www.bilo-data.de. Zugegriffen: 1. Nov. 2019.

Stein, A., \& Bock-Famulla, K. (2017). Kita-Qualität steigt, hängt aber vom Kreis ab. https://www. bertelsmann-stiftung.de/de/themen/aktuelle-meldungen/2017/august/kita-qualitaet-steigt-haengtaber-vom-kreis-ab/. Zugegriffen: 1. Nov. 2019.

Strehmel, P. (2015). Leitungsfunktion in Kindertageseinrichtungen: Aufgabenprofile, notwendige Qualifikationen und Zeitkontingente. In S. Viernickel, C. Preissing, J. Bensel, G. Haug-Schnabel, P. Strehmel \& K. Fuchs-Rechlin (Hrsg.), Qualität für alle: wissenschaftlich begründete Standards für die Kindertagesbetreuung (S. 131-252). Freiburg im Breisgau: Herder.

Strehmel, P., \& Ulber, D. (2014). Leitung von Kindertageseinrichtungen: Eine Expertise der Weiterbildungsinitiative Frühpädagogische Fachkräfte (WiFF). München: Deutsches Jugendinstitut.

Tietze, W. (Hrsg.). (1998). Wie gut sind unsere Kindergärten? Eine Untersuchung zur pädagogischen Qualität in deutschen Kindergärten. Neuwied: Luchterhand.

Tietze, W. (2008). Qualitätssicherung im Elementarbereich. Qualitätssicherung im Bildungswesen: eine aktuelle Zwischenbilanz (S. 16-35).

Tietze, W., \& Lee, H.-J. (2009). Ein System der Evaluation, Verbesserung und Zertifizierung pädagogischer Qualität von Kindertageseinrichtungen in Deutschland. In K. Altgeld \& S. Stöbe-Blossey (Hrsg.), Qualitätsmanagement in der frühkindlichen Bildung, Erziehung und Betreuung: Perspektiven für eine öffentliche Qualitätspolitik (S. 43-62). Wiesbaden: Springer VS.

Tietze, W., \& Viernickel, S. (Hrsg.). (2016). Pädagogische Qualität in Tageseinrichtungen für Kinder. Ein Nationaler Kriterienkatalog. Weimar, Berlin: Verlag das Netz.

Tietze, W., Becker-Stoll, F., Bensel, J., Eckhardt, A. G., Haug-Schnabel, G., Kalicki, B., Keller, H., \& Leyendecker, B. (Hrsg.). (2013a). Nationale Untersuchung zur Bildung, Betreuung und Erziehung in der frühen Kindheit: (NUBBEK). Weimar, Berlin: Verlag das Netz.

Tietze, W., Lee, H.-J., Bensel, J., Haug-Schnabel, G., Aselmeier, M., \& Egert, F. (2013b). Pädagogische Qualität in Kindertageseinrichtungen und Kindertagespflegestellen. In W. Tietze, F. Becker-Stoll, 
J. Bensel, A. G. Eckhardt, G. Haug-Schnabel, B. Kalicki, H. Keller \& B. Leyendecker (Hrsg.), Nationale Untersuchung zur Bildung, Betreuung und Erziehung in der frühen Kindheit: (NUBBEK). Weimar, Berlin: Verlag das Netz.

Tietze, W., Roßbach, H.-G., \& Grenner, K. (2005). Kinder von 4 bis 8 Jahren: Zur Qualität der Erziehung und Bildung in Kindergarten, Grundschule und Familie. Weinheim, Basel: Beltz.

Vandell, D. L., \& Wolfe, B. (2000). Child care quality: Does it matter and does it need to be improved? Institute for Research on Poverty. https://www.irp.wisc.edu/publications/sr/pdfs/sr78.pdf. Zugegriffen: 10. Okt. 2019.

Vermeer, H.J., van IJzendoorn, M., Cárcamo, R. A., \& Harrison, L. (2016). Quality of child care using the environment rating scales: a meta-analysis of international studies. International Journal of Early Childhood, 48, 33-60.

Viernickel, S. (2008). Qualitätskriterien und -standards im Bereich der frühkindlichen Bildung und Betreuung. Remagen: Ibus.

Viernickel, S., Nentwig-Gesemann, I., Nicolai, K., \& Schwarz, S. (2013). Schlüssel zu guter Bildung, Erziehung und Betreuung: Bildungsaufgaben Zeitkontingente und strukturelle Rahmenbedingungen in Kindertageseinrichtungen. Forschungsbericht. Berlin: Der Paritätische Gesamtverband.

Watamura, S.E., Phillips, D. A., Morrissey, T. W., Mc Cartney, K., \& Bub, K. (2011). Double jeopardy: Poorer social-emotional outcomes for children in the NICHD SECCYD experiencing home and childcare environments that confer risk. Child Development, 82, 48-65. 